\title{
PERSPECTIVES
}

\section{Can cell systems biology rescue drug discovery?}

\section{Eugene C. Butcher}

Abstract | The focus of innovation in current drug discovery is on new targets, yet compound efficacy and safety in biological models of disease - not target selection - are the criteria that determine which drug candidates enter the clinic. We consider a biology-driven approach to drug discovery that involves screening compounds by automated response profiling in disease models based on complex human-cell systems. Drug discovery through cell systems biology could significantly reduce the time and cost of new drug development.

The perceived failure of current drug discovery has generated widespread concern, and several divergent opinions about the problem and its potential solutions. Horrobin ${ }^{1}$ has gone so far as to liken current drug discovery to an intellectually absorbing but meaningless game, divorced from the reality of medicine. A disconnection between pharmaceutical research and successful new drug discovery is indeed apparent. Far from the explosion of new drugs predicted to follow the sequencing of the human genome, the overall rate of new drug approvals has failed to keep pace with ever-increasing spending on pharmaceutical research ${ }^{2-6}$. Even more worrisome is the rate of approval of drugs against new targets (molecules not the targets of previous drugs): over the past decade, the entire industry has averaged only two to three small-molecule drugs against such 'innovative' targets per year ${ }^{7,8}$. Why is there not more innovation in drug therapies? What has gone wrong?
Since the early successes of compound screening against isolated molecular targets in the 1970s, the industry has directed more and more of its research towards target-directed drug discovery. Target-based screening was initially used to improve the drug-like properties and selectivity of pharmacologically active products; indeed, it has been very successful when applied to wellvalidated targets (that is, targets of known drugs). Some commentators promised that sequencing the human genome would generate a wealth of new targets, and the hope of 'genes-to-drugs' was embraced wholeheartedly by the industry in the 1990s. When the drug discovery process moved beyond historical targets, however, it became apparent that the target-directed approach was flawed: without solid biological validation, target-based drug discovery has proven very disappointing ${ }^{3,9}$. Although a number of underlying problems with the current paradigm have been highlighted, here we focus on two that seem particularly crucial to the rate of innovation. First, target validation (independent of an inhibitory drug) could be fruitless. Current mouse-genetics-focused methods of target validation cannot reliably predict human biology; and even if a model is predictive of human target biology, target biology cannot reliably predict drug biology. One instance of this is the failure of antagonists of the neurokinin 1 (NK1) receptor as analgesics ${ }^{10}$, but a recent review reminds us, with many examples, that mice are not men ${ }^{11}$. Second, the target-specific approach is exceedingly slow: only one target can be screened at a time, which creates an intrinsic bottleneck that, especially for novel targets of questionable validity, is certainly a barrier to rapid progress.

The failure of the target-based genes-todrugs paradigm to live up to its promise has led to a disheartening atmosphere of caution, and the industry is increasingly turning away from innovative targets to focus on 'safe' programmes - improving on existing drugs or developing 'me too' drugs against established blockbuster targets ${ }^{6,12}$. Even venture capitalists seem resistant to venturing beyond safe programmes. This atmosphere does not bode well for an industry whose health ultimately depends on the development of novel therapies for unmet medical needs; real advances in medicine must include the generation of drugs that act through novel mechanisms. Clearly, it is time to re-examine the basic premise of the current drug discovery paradigm. But what alternative is there to target-directed discovery?

\section{A cell systems biology approach?}

Here we explore the potential implications of a drug discovery paradigm in which complex biological responses are used directly to screen for and select lead candidates. In a way, this can be considered a return to the biology-directed drug discovery that gave us the earlier generations of medicines that underlie many modern-day drugs - but accelerated through the application of advances in human cell models of disease (FIG. 1). Recent studies suggest that primary human cell systems can be designed to model many aspects of disease biology ${ }^{13,14}$, and robust, automated assays can be engineered to detect and discriminate a surprising breadth of disease-relevant pathways and mechanisms ${ }^{14,15}$. These assays benefit from principles of systems biology, and mimic the complexity of disease processes by incorporating multiple cell types and activating multiple pathways together. They represent a practical approach to systems biology for drug discovery, because they directly 


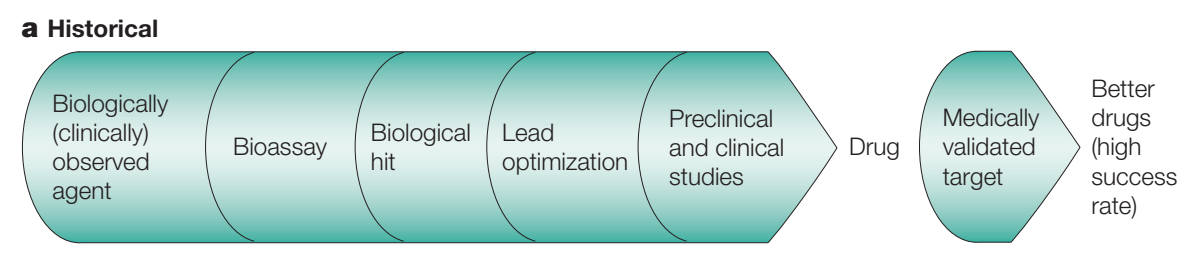

b Current target-based discovery

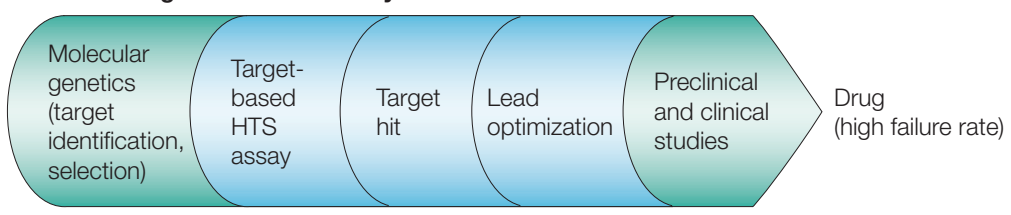

C Proposed cell systems biology approach

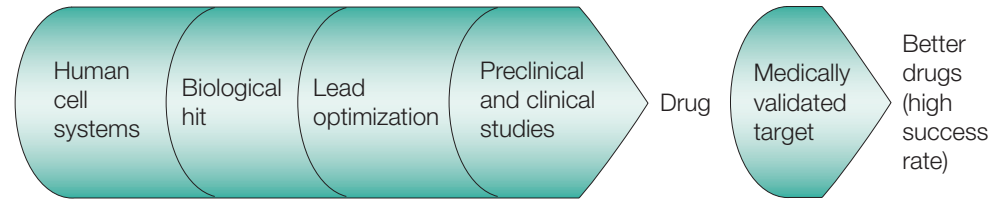

Figure 1 | Drug discovery paradigms. a | Historically, there has been considerable success in developing drugs to the targets of natural medicines or of previous drugs. This made the current targetdirected discovery approach (b) seem attractive, but in the past this approach benefited from a degree of target prevalidation that is beyond the reach of current drug-independent methods. Not surprisingly, drug candidates developed against less well-validated targets (for example, genome-derived targets, often validated genetically in animal and gene-expression studies) have a had a much lower success rate. c | The cell systems biology approach again places biology first, and applies biological insights throughout the discovery process: it identifies hits and optimizes leads on the basis of their elicited biology in complex cell systems that model diverse active cells, pathways and networks in disease states of interest. In this approach, the drug candidate itself, not a target gene or protein, is used for validation. Similar preclinical studies (animal models; toxicology; absorption, distribution, metabolism and excretion) and clinical studies must be applied to validate drug candidates in each of the approaches. Stages in discovery that are directed by biological input are indicated in green.

measure disease-relevant cellular responses without the need for complex in silico models whose application to predicting human cell responses, except in proscribed settings, is many years in the future. We compare and contrast a drug discovery programme based on such cell systems biology with the current target-based drug discovery paradigm.

What might be the advantages of putting biology first? First, there is ample precedent for this approach. Indeed, as mentioned above, most of today's successful drugs are the chemical descendents of natural products or bioactive agents that were originally selected for their biological effects - often their medicinal properties, but in some cases their effects on animal physiology ${ }^{18}$ (FIG. 1). Second, biology is the gatekeeper to the clinic. An orally available compound that is effective and safe in human cell assays and animal models of disease, and which has good pharmacological and pharmaceutical properties, can enter clinical trials in the absence of knowledge of the biochemical target. Given instead a potent inhibitor of a defined but novel molecular target, even if the target has been extensively validated in animal studies, regulatory agencies will appropriately ask for biological evaluation of the drug candidate itself. Validation of the drug, not the target, is required. Third, biological assays, and especially cell systems engineered to incorporate disease-relevant complexity, can cast a much wider net than target-by-target approaches. Selection of possible drug targets from genes is in practice limited to certain known target classes (for example, G-protein-coupled receptors (GPCRs) and enzymes). By these criteria, there are only an estimated 3,000 'druggable' genes in the human genome (of which only a fraction would or could be therapeutic targets $)^{8}$. However, many potent biological agents do not in fact bind to these predictably druggable target classes: for example, the immunosuppressive drugs FK506 and cyclosporin act as protein-protein-binding adaptors to trigger and stabilize interactions of their cognate immunophilins with calcineurin. Another example, this time from random compound screening, is an inhibitor of the heterodimeric integrin leukocytefunction-associated antigen 1 (LFA1): this small molecule acts allosterically to inhibit integrin activation ${ }^{16}$. The number of potential small-molecule intervention sites, although unknowable, is therefore likely to be quite large. Moreover, Kubinyi ${ }^{5}$ has pointed out that many drugs, including many non-steroidal anti-inflammatory drugs (NSAIDs) and most psychotropic drugs (such as clozapine), owe their unique medicinal qualities to simultaneous inhibition of more than one molecular target. Kubinyi ${ }^{5}$ argues that each unique target combination could therefore be considered a pharmacologically relevant 'target' itself. According to this criterion, the number of targets that can be accessed by complex biological screens might be essentially unlimited.

In addition to capturing extensive 'target space', cell systems biology assays can also be designed to capture aspects of biology of a broader range of diseases than standard cellular assays, or even than typical animal studies or clinical trials. (This is not to say that in vitro assays can ever model the biology of a disease as well as a focused clinical trial, or that the biology of all diseases can be modelled (BOX 1). Rather, it is to say that cell systems models can be designed to 'scan' a wide range of biology more rapidly and cost effectively than in vivo studies.) Just as conventional cellular assays focus on evaluating a single pathway or response, animal studies and clinical trials generally evaluate effects in a specific disease state. Lead candidates are, of course, subjected to additional biological assays, but often these are constrained by programmatic or cost concerns - and the pursuit of simultaneous clinical trials in several diseases is clearly problematic from an economic perspective.

These approaches therefore typically encompass limited biology by design. This limitation can be a real impediment to pharmaceutical success, because many drug candidates fail due to unpredicted activities that might have been detected by more broadly based biological screens; and a surprising number of marketed drugs owe their success to unexpected biological effects. Observations of the antidepressant and antipsychotic activity of early antihistamines - which led to modern psychopharmaceuticals ${ }^{17}$, the discovery of the immunosuppressant activity of the antifungal agent cyclosporin, and the surprising diversification of derivatives of the antibiotic sulphanilamide as therapies for diabetes and hypertension $^{3}$ - provide classic examples of the dependence of drug discovery on serendipity and opportunism, and of the interplay of chemistry and broad clinical/biological observations. In contrast to the necessarily 


\section{Box 1 | Target-centric drug discovery or a cell systems biology approach?}

Issues facing target-centric drug discovery

- Not working as well as hoped as a path to innovative medicines.

- Limits discovery to known molecular targets or target classes and 'perceived modes of action'.

- Fosters a mindset of caution - because most obviously 'druggable' targets have not been validated biologically, the industry (and even venture-capital firms) are increasingly focusing on 'me too' drug discovery.

- Front-loads the time and expense of biological validation (new targets must be validated prior to screening; then drug candidates need to be validated again).

- Restricts drug discovery to an unreasonably small sampling of 'target space'.

Features of a cell systems biology approach

- Assays large number of potential drug targets simultaneously.

- Induced biology informs initial prioritization and hit selection.

- Encompasses extensive range of disease-relevant biology (though not comprehensive).

- Can be designed to 'scan' multiple disease models and mechanisms ('therapeutic-area scanning').

- Limited to therapeutic areas (cell types and networks) amenable to simulation in tissue culture models.

- Although much of inflammation, metabolism and cell-cycle control/cancer biology can be modelled, neuropharmacology, for example, will be difficult to incorporate in a cell systems biology approach, as would whole-organism-level feedback mechanisms.

- Limited to targets and mechanisms expressed in the cultured cell types.

- Could improve quality of hits (smaller libraries, prequalified for drug-like qualities).

- Eliminates time and expense of target validation prior to screening (drug candidates, not targets, are validated in animal models and patients).

- Quantitative system-response profiles drive lead optimization.

- Validated drug candidates used to identify molecular targets. focused nature of animal studies and human trials, inexpensive complex cell systems can be designed to incorporate the biology of several physiological and disease states and multiple pathological environments, which will increase the chances of discovering unexpected therapeutic opportunities.

As an example, using four complex human cell systems, Kunkel et al. ${ }^{14,18}$ were able to cover many of the pathways and responses involved in asthma ( $\mathrm{T}_{\mathrm{H}}$ 2-mediated inflammation), autoimmune disease and arthritis ( $\mathrm{T}_{\mathrm{H}} 1$-mediated inflammation), transplantation (T-cell driven) and cardiovascular disease-related (monocyte and endothelial cell-driven) inflammatory responses. The four systems (cell-environment combinations) used in the study comprised primary human endothelial cells in complex $\mathrm{T}_{\mathrm{H}} 1$ (system 1) or $\mathrm{T}_{\mathrm{H}} 2$ (system 2 ) cytokine environments, or endothelial cells co-cultured with human peripheral blood mononuclear cells (which comprise multiple cell types, including $\mathrm{T}$ cells, monocytes, natural killer cells and so on) in settings of Toll receptor/ monocyte-driven (system 3) or of T-cellreceptor-driven (system 4 ) signalling cascades (FIGS 2,3). Readouts were selected for disease significance and information content, and both the readouts and the systems themselves were chosen on the basis of efforts to identify a minimal assay set that could detect and differentiate between most immune- or inflammation-modulating agents active the modelled biologies, and clustering of the combined systems profiles (as well as effects of the compounds on clinically relevant readout parameters), can provide rapid insights into mechanisms of action, and into potential therapeutic significance. It is likely that more restricted parameter sets can be selected for primary screening that would retain sensitivity to diverse mechanisms; but for the purposes of our discussion here, we consider a screening application of the automated cell systems assays as published, which evaluate 31 readouts in total from the four inflammatory model systems described (seven to ten protein measurements per cell system $)^{14,18}$. This combined systems assay detects and discriminates more than 100 mechanistically distinct immunomodulatory drugs or agents, including not only anti-inflammatory drugs but also a surprising array of drug targets and pathways for other diseases, such as cancer and metabolic disorders ${ }^{14}$ (FIGS 2,3). In principle, it would also detect any unknown pathway or target that interacts significantly with these known mechanisms. Adding more cell systems could further expand disease and pathway coverage. In addition to enhancing the potential of screening efforts as emphasized here, cell systems biology-based 'therapeutic-area scanning' could help expand the opportunities for target-based leads, allowing in these diseases. This ensures very broad coverage of the therapeutic 'target space' of interest. The profile generated by a given compound serves as a signature of its function in

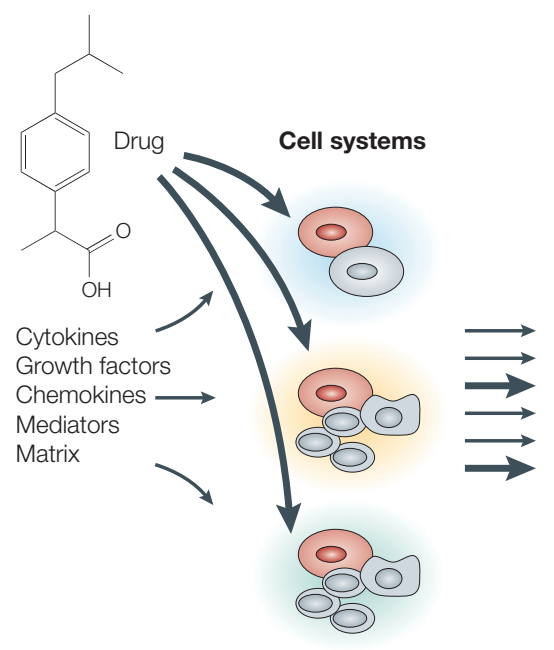

\section{Selected protein}

readouts
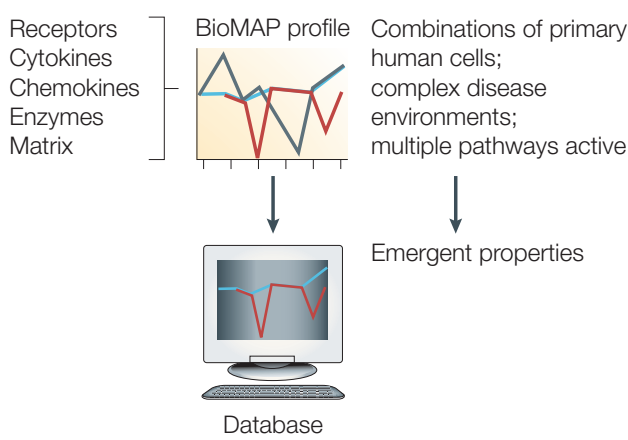

Figure 2 | Cell systems biology for drug discovery. In the cell systems biology approach, diseaserelevant complexity is engineered into cell 'systems' by combining different cell types together, and by activating multiple pathways simultaneously to elicit network regulation and emergent properties. For example, a cell systems approach to inflammatory diseases (see FIG. 3) includes combinations of endothelial cells and blood mononuclear cells, and complex cytokine or immune-stimulating environments. Readouts used to measure system responses typically include proteins or other cellular constituents, or properties selected for disease relevance (for example, cytokines, growth factors or adhesion receptors the ultimate mediators of cellular function and of disease) and for responsiveness to diverse environmental or pharmacological inputs (information content). The combination of cells, stimuli and readouts in each system, and the combination of systems assayed together is designed to detect and discriminate as many known disease-modulating agents and drugs as possible with a minimal set of measurements. 
a Multiple cell and environment 'system'

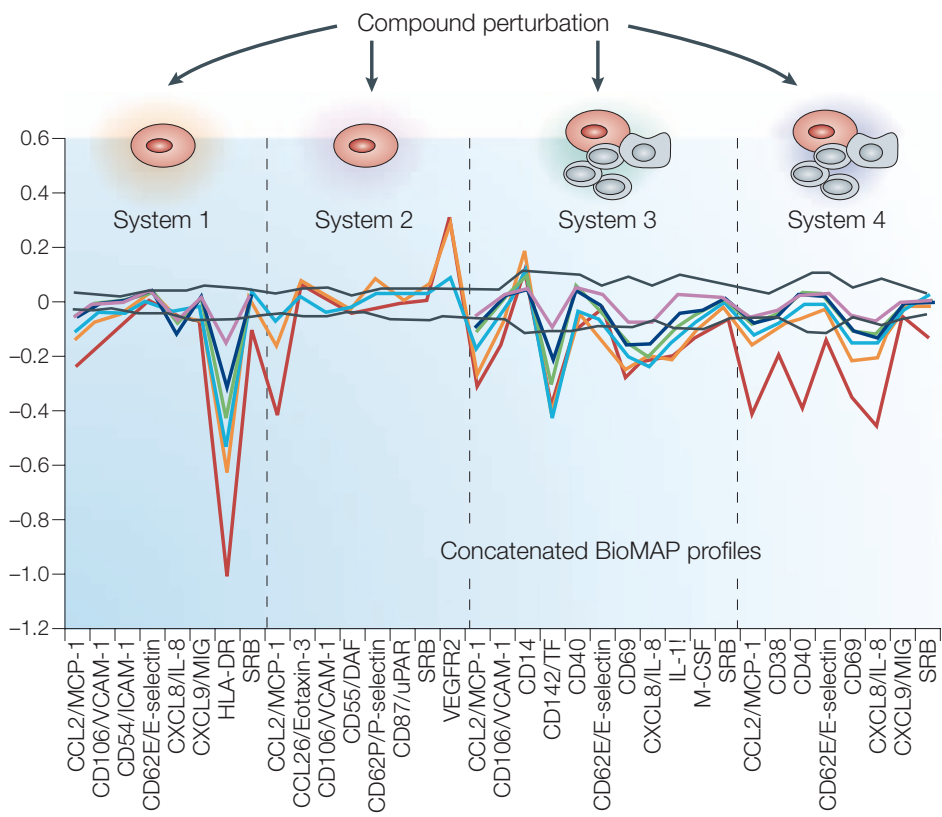

e Prioritization: classification by biological function
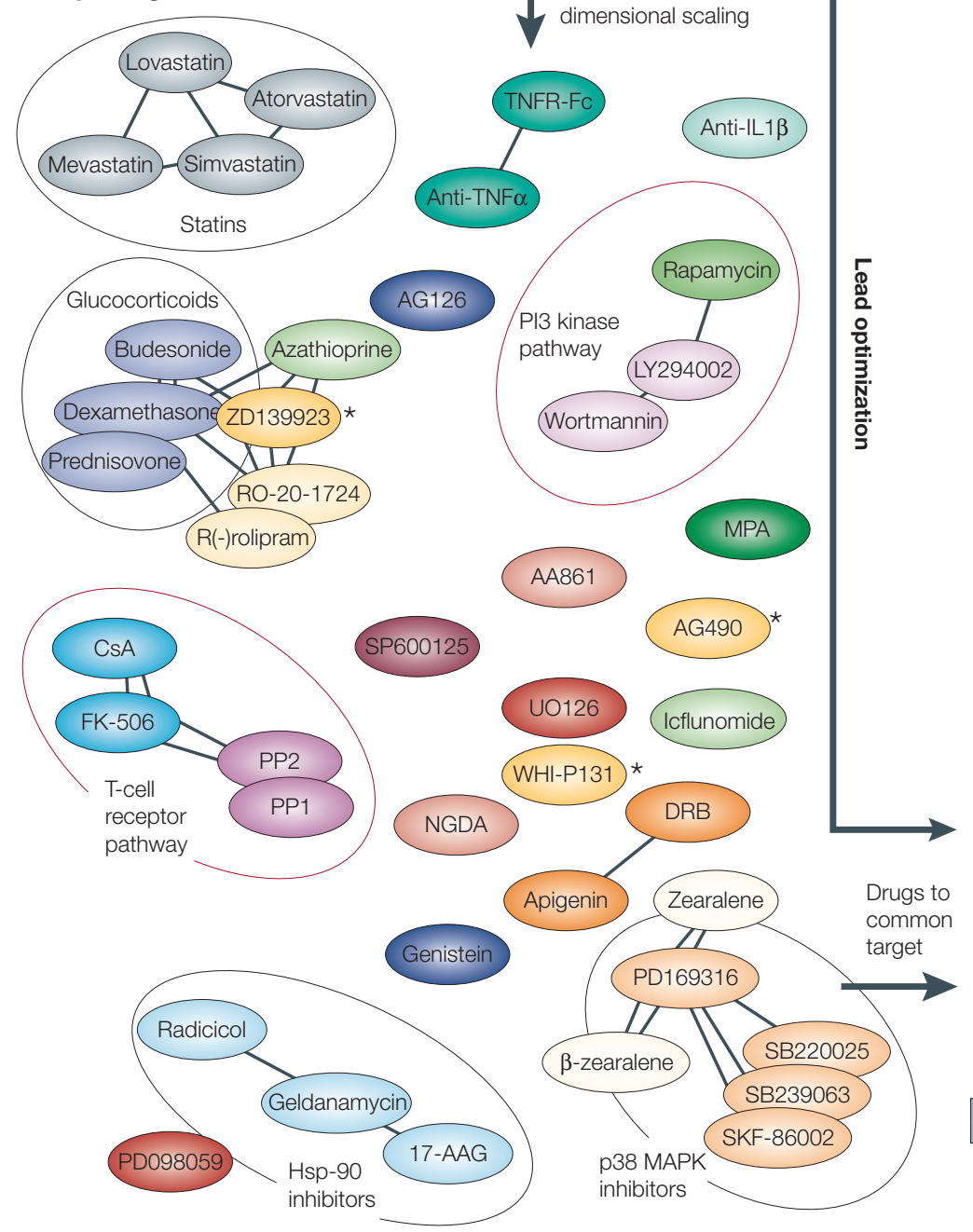

Preclinical candidates

Pairwise correlation and multidimensional scaling Y b Broad coverage of 'target space'

Sensitive to many pathways and mechanisms, for example:

Inflammation/immunomodulation:

Cyclophilin; FKBP; glucorticoid receptor; cyclooxygenase; methotrexate; DOODH; IL-10; IL-RA; mTOR; TNF- $\alpha$ antagonists azathioprine; p38 MAPK; JAK; Lck; MEK; JNK; IMPDH; CB1, $\mathrm{CB} 2$ receptor; bradykinin receptor; antioxidants; NF- $\mathrm{KB}$ activation; lipooxygenase; adenosine receptor; thromboxane receptor; leukotriene receptors; phospolipases, DAG lipase

\section{Cardiovascular:}

ACE; ACAT; L-type $\mathrm{Ca}^{2+}$ channel; $\beta$-adrenergic blocker; HMG-CoA reductase; guanylyl cyclase, NOS; angiotensin II receptor; endothelin receptor; PAR receptors

\section{Screening Cancer/other:}

Histone deacetylase; HSP90; proteasome; PI3 kinase, AKT; CDK2; RAR/RXR; c-RAF, AKT; PKC; VEGFR, PDGFR;

microtubule stabilizers; antivirals

\section{Metabolism:}

PPAR $\gamma / \delta$; LXR; oestrogen receptor; androgen receptor; GSK-3 $\beta$

\section{Asthma/allergy:}

$\mathrm{H}_{1}$ receptor; $\beta_{2}$ agonist; mast-cell stabilizers; PDE4; PAF receptor; Syk

\section{Experimental:}

ROCK; adenylyl cyclase; HOX; BTK, RKR, CAPK; IKK, CK2; CaMKII; PKA, PKG

\section{d Chemical structures}<smiles>Nc1nccc(-c2c(-c3ccc(F)cc3)ncn2C2CCNCC2)n1</smiles><smiles>COc1nccc(-c2c(-c3ccc(F)cc3)ncn2C2CCC(O)CC2)n1</smiles><smiles>O=[N+]([O-])c1ccc(-c2nc(-c3ccc(F)cc3)c(-c3ccncc3)[nH]2)cc1</smiles><smiles>CS(=O)c1ccc(-c2nc(-c3ccc(F)cc3)c(-c3ccncc3)[nH]2)cc1</smiles>

c Structure-activity relationships
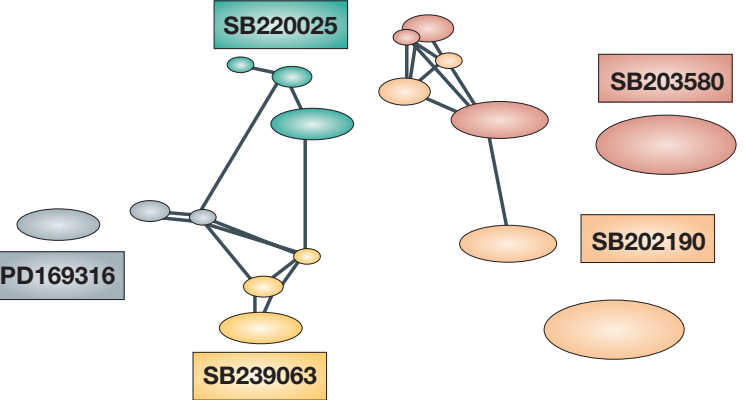

SB202190 
4 Figure 3 | Cell systems biology as applied to drug discovery in inflammation. $\mathbf{a , b} \mid$ Screening: four complex systems incorporating complex $\mathrm{T}_{\mathrm{H}}{ }^{1-\text {, }}$ $T_{H}$ 2-, Toll receptor/monocyte-driven and T-cellreceptor-driven environments are interrogated with compounds or drugs, and the effects on the levels of selected protein readouts are determined, generating a profile that serves as a multi-system signature of the function of each test agent; the assay is sensitive to a wide array of known drugs and pathways, and can therefore be used to screen drug-like compound libraries for compounds affecting any of these mechanisms (as well as many unknown mechanisms) ${ }^{14}$. c| Lead optimization through structure-activity relationships (SARs): statistical measures of profile similarity (that is, do agents induce the same multi-system response?) can be used to cluster drugs by function, and multi-dimensional scaling is used to generate simplified graphical representations of their functional relationships with each other ${ }^{14}$. Clustering of activity profiles from lead chemical series can define compound-specific SARs for lead optimization (lower right; different analogues are colour coded; circle size reflects concentration). In the example shown ${ }^{18}$, two functional activity classes were defined among structurally related p38 mitogen-activated protein kinase (MAPK) inhibitors. d | Chemical structures of the p38 MAPK inhibitors profiled in c. e | Prioritization of hits and leads: prioritization of active compounds is aided by the effects on the (clinically relevant) readouts used, and by benchmarking against a database of activity profiles of known drugs and immunomodulators (illustrated in the lower left; drugs are coloured by mechanistic class, and lines or 'edges' between drugs reflect a measure of statistical significance $)^{14,18}$. Drugs specific for a common target (circled in black), or for targets in a common pathway (circled in red), cluster together, but compounds with different off-target activities are readily detected (for example, the profiles of three Janus kinase (JAK) inhibitors with known secondary activities; asterisks).

compounds generated during lead optimization to be tested for effects in multiple disease areas. It could also be used to search for novel indications for shelved drugs.

\section{Comparison with target-based screens}

As a first approximation, the frequency of hits in a compound-screening campaign is proportional to the number of compounds screened and the number of therapeutically relevant targets assayed per screen. A typical high-throughput screening (HTS) programme can evaluate a million compounds against one target, generating $10^{6} \times P_{h}$ initial hits (where $P_{h}$ is the probability that a given compound is a hit on a given target). A cell systems biology screen as considered here might reasonably evaluate 500 compounds per week, per robotic system (although this too would be scalable), but encompasses sensitivity to hundreds of potential targets (FIGS 2,3). If we conservatively estimate that 100 small-molecule intervention sites might be of therapeutic interest, hits would be expected at the rate of $500 \times 100 \times$ $P_{h}$ or $5 \times 10^{4} \times P_{h}$ per week. The number of hits is in this case a function of time as well, and can continue until the applicable compound library is fully screened.

Moreover, the cell systems approach might generate 'better' hits. One of the most obvious advantages of screening fewer compounds in assays sensitive to many potential intervention mechanisms is the opportunity to preselect compounds for drug-like qualities. (One reason for the limited success of many ultra-HTS efforts is the poor or nondrug-like quality of many of the compounds in large combinatorial chemistry libraries ${ }^{3,4}$. By contrast, when hundreds instead of millions of compounds are screened per week, compounds can be qualified for desirable characteristics ${ }^{19,20}$ prior to screening.) Certainly, compounds selected in cell systems assays will be soluble, cell permeable when needed, non-cytotoxic and will have desirable initial biological effects. Such compounds could therefore represent relatively advanced hits, and could more frequently be amenable to rapid optimization and conversion to lead candidates than many hits from less selective libraries.

Equally important, but less easy to predict, is the advantage and potential time savings associated with the biological information inherent in the activity profiles generated in the screening process. Published studies show that cell systems profiling in models of inflammatory biology can help in assigning priority to compounds. Furthermore, such profiles can suggest potential therapeutic applications according to their effects on therapeutically significant cell system responses, such as regulation of inflammatory cytokine or cell-trafficking molecule expression; or by correlation of compound activity profiles (and therefore function) with those of known therapeutics ${ }^{14,18}$.

Perhaps the most significant difference between conventional and cell systems-based approaches, however, is the time and effort required to sample a reasonable amount of new 'target space'. Admitting that one cannot $a$ priori know which genes encode useful therapeutic targets, the target-focused model requires that each new target be selected and validated on the basis of expression and biological function. Increasingly, such validation relies on genetic or antibody approaches to animal models of disease. The relevance of these models is often questioned, but even if they are accepted as being predictive, the inescapable consequence of this approach is that substantial effort, expense and time is devoted to every target before it is even screened.

It has been estimated that the aggregate effort devoted to pharmaceutical target identification and validation (including the many failed efforts) amounts to 3 years and US\$390 million for each new drug reaching the market ${ }^{2}$ (summarized in TABLE 1). Then, after leads are generated from target-based hits, the candidate drugs must themselves be validated again in human cell and animal disease models. The time and cost involved provides a practical limit on the number of targets that can be evaluated and screened. By contrast, cell systems biology assays can be engineered to incorporate many diseaserelevant pathways and hundreds of known target responses by design - because they embody so many potential targets, there is no need to develop new assays every week to satisfy the hunger of HTS robots. The same cell systems can be assayed indefinitely until a sufficient number of desirable leads are generated in the therapeutic areas being modelled. Most importantly, in this case, validating studies are applied to the resulting lead compounds themselves.

How would a cell systems biology paradigm for drug discovery differ from conventional high-throughput cell-based screens? Although cell systems assays endeavour to incorporate the biological complexity characteristic of cell- and (to the extent possible) tissue-level disease processes, conventional cell-based screening (and even most forward chemical genetic efforts) try to restrict the complexity of assays, focusing on an individual cellular response in a simplified environment. A typical example is the widespread use of engineered reporter cell lines for specific signalling pathways. Such pathway responses can screen for effects on targets integral to a linear pathway, but by design exclude complex pathway-pathway and cell-cell interactions that allow cell systems biology approaches to encompass such a breadth of biology. (Even 'high content' cell assays, which measure multiple phenotypic or biochemical cellular outputs simultaneously, are typically applied to cell lines in simplified environments, to avoid the complexity of disease settings and primary cells ${ }^{21}$.) Combinations of pathway screens can extend the coverage, and can even be used to classify compound activities according to the sets of pathways affected. However, simplified cellular assays by their very nature preclude the emergent properties that characterize complex cell systems responses 


\begin{tabular}{|c|c|c|c|}
\hline $\begin{array}{l}\text { Discovery/ } \\
\text { development } \\
\text { phase }\end{array}$ & $\begin{array}{l}\text { Current } \\
\text { paradigm }\end{array}$ & $\begin{array}{l}\text { Cell systems } \\
\text { biology }\end{array}$ & Assumptions \\
\hline Target ID & $\begin{array}{l}\$ 165 \mathrm{M}, \\
\text { over } 3 \text { years }\end{array}$ & $\mathrm{N} / \mathrm{A}$ & No target ID/validation before screening ${ }^{\ddagger}$ \\
\hline $\begin{array}{l}\text { Target } \\
\text { validation }\end{array}$ & $\begin{array}{l}\$ 205 \mathrm{M} \\
\text { over } 3 \text { years }\end{array}$ & $\mathrm{N} / \mathrm{A}$ & \\
\hline Screening & $\begin{array}{l}\$ 40 \mathrm{M}, \\
\text { over } 0.4 \text { years }\end{array}$ & $\begin{array}{l}\$ 30 \mathrm{M}, \\
\text { over } 1 \text { year }\end{array}$ & $\begin{array}{l}\text { Reduced cost from screening smaller } \\
\text { libraries and lack of need to develop } \\
\text { target-specific assays }\end{array}$ \\
\hline Optimization & $\begin{array}{l}\$ 120 \mathrm{M} \text {, } \\
\text { over } 2.7 \text { years }\end{array}$ & $\begin{array}{l}\$ 100 \mathrm{M} \text {, } \\
\text { over } 2.1 \text { years }\end{array}$ & $\begin{array}{l}\text { Reduced cost from preselection of } \\
\text { compounds for drug-like characteristics }\end{array}$ \\
\hline Preclinical & $\begin{array}{l}\$ 90 \mathrm{M}, \\
\text { over } 1.6 \text { years }\end{array}$ & $\begin{array}{l}\$ 90 \mathrm{M}, \\
\text { over } 1.6 \text { years }\end{array}$ & $\begin{array}{l}\text { Enhanced cost for extensive cell systems } \\
\text { profiling, offset by reduced cost from } \\
\text { improved selection of animal models }\end{array}$ \\
\hline $\begin{array}{l}\text { Drug } \\
\text { target ID }\end{array}$ & $N / A^{\ddagger}$ & $\$ 10 M^{\ddagger}$ & $\begin{array}{l}\text { Estimated cost for identification of targets } \\
\text { of validated preclinical leads (optional) }\end{array}$ \\
\hline Clinical & $\begin{array}{l}\text { \$260M, } \\
\text { over } 7 \text { years }\end{array}$ & $\begin{array}{l}\text { \$260M, } \\
\text { over } 7 \text { years }\end{array}$ & $\begin{array}{l}\text { Possible reduction in cost from improved } \\
\text { indication selection and biomarker } \\
\text { identification (not modelled) }\end{array}$ \\
\hline Total & $\begin{array}{l}\$ 880 \mathrm{M}, \\
\text { over } 14.7 \text { years }\end{array}$ & $\begin{array}{l}\$ 490 \mathrm{M}, \\
\text { over } 11.7 \text { years }\end{array}$ & \\
\hline \multicolumn{4}{|c|}{ 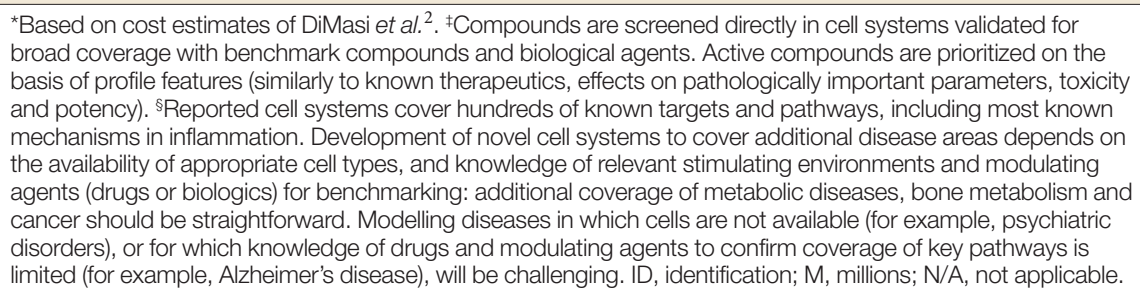 } \\
\hline
\end{tabular}

- and which provide much of the diseaserelevant biological insights that help prioritize hits and leads. One way to deal with this limitation, of course, is to apply cell systems approaches to prioritize hits from more conventional cell- or target-based HTS.

\section{Challenges to the approach}

Several challenges to the cell systems biology approach to drug discovery need to be addressed (BOX 1). One intrinsic issue is the availability of primary cell types, and the ability of primary cells alone or in combination to reflect disease biologies and mechanisms of interest. Although many diseases, from inflammation and metabolic disorders to cancer biology, can be addressed, cell systems modelling of complex neural networks underlying neuropsychiatric disorders would seem beyond current art, for example. (Simple cultured neuron models could well prove sufficient for the functional classification of neuropsychiatric drugs, however, including the prediction of potential utility in depression versus psychoses ${ }^{22}$.) Similarly, complex organism-wide signalling that is mediated by networks of hormones can only be modelled rather crudely, by using systems comprising responding cell types in environments with different hormone levels. Moreover, although the range of primary human cells available commercially is surprising - from keratinocytes to bronchial epithelium, smooth muscle and even neuronal precursors - the list is not comprehensive.

Additional challenges are presented by lead optimization and target identification. In target-based approaches, target binding or inhibition is used not only for screening but also to define structure-activity relationships (SAR) for optimization of hits. Historically, of course, lead optimization was pursued successfully without target-based optimization - even in the days when animal physiology was the only 'readout ${ }^{3,17,23}$. Moreover, recent studies indicate that cell systems profiling can also be used to define SAR and to drive efficient optimization ${ }^{18}$. Biology-driven lead optimization also has the advantage that it monitors not only changes in potency, but also off-target or altered-target specificity as revealed by alterations in profile shape or functional signatures ${ }^{14,18}$. In model studies of chemically related p38 mitogen-activated protein kinase (MAPK) inhibitors, for example, complex cell systems responses readily identified the most effective and least cytotoxic variants in an SAR series. More importantly, the profiles that were generated defined a functional signature common to the p38 MAPK inhibitors, readily identified the offtarget activity of SB20358024, and correlated this activity to modifications in a particular side chain ${ }^{18}$. The reproducibility, information content and throughput of biosystemsresponse profiling can therefore substitute for conventional target-based SAR, and can directly drive medicinal chemistry efforts.

Nonetheless, after a lead compound or new chemical entity (NCE) proves effective and safe in vivo, an understanding of the target(s) involved will be of interest both for basic research reasons, and for developing second-generation drugs (paralleling the original use of target-based methods). Identification of the now-validated drug target might be the ideal application for global proteomic and gene-expression analyses, which, in conjunction with cell systems biology profiles to point to particular pathways, can help identify the precise site of action of drugs much more rapidly than conventional approaches. (This NCE-to-target approach, historically the province of pharmacology, is now seeing a resurgence of interest as 'forward chemical genetics.) The effort and time required to identify the target of a wellcharacterized clinical or late-stage preclinical lead could be on the same order as that typically expended for validation (or rejection) of a putative screening target; in this case, however, the effort is expended not before the fact on many targets (most of which are rejected), but only after there is convincing proof of concept in the form of an effective drug-like compound.

\section{Implications and conclusion}

What are the economic implications of a drug discovery paradigm driven by cell systems biology? How might the time and cost to new drug launch compare with those reported for target-based discovery? Using the widely cited summary of drug development costs from DiMasi², TABLE 1 compares a cell systems approach with that of conventional targetbased discovery. As outlined in TABLE 1, it is reasonable to assume modest reductions in the cost of screening (because there is no need to establish a different assay system each week or two) and lead optimization (based on screening more drug-like compounds). Medicinal chemistry costs and efforts can be applied to maximum advantage by therapeutic-area scanning and programme crossfertilization. At the same time, preclinical animal models, predictive toxicology studies and clinical trial costs might benefit from surrogate markers and improved clinical 
indication selection; however, because cell systems biology can be used to help prioritize target-based leads as well, we can assume that these expenses will be comparable. Obviously, the real economic benefit lies in the elimination of target identification and validation prior to compound screening. This alone has the potential to reduce the time required for innovative drug discovery by years and reduce costs by hundreds of millions of dollars. A major reduction in drug development costs could also increase the profitability of non-blockbuster drugs (drugs with less than US\$1-billion potential sales per year), thereby providing incentives for the industry to address a wider range of diseases ${ }^{25}$.

With the current uncertainty in the pharmaceutical industry - from political concerns about drug pricing to legal ramifications of the Vioxx fiasco - can the industry afford to explore a new paradigm for drug discovery? Perhaps a more relevant question would be whether it can afford not to. The gears in the engine of drug discovery spin exceedingly rapidly these days, but the lack of satisfactory forward motion suggests a fundamental flaw in the engine's design. The modest proposal here - not to discard but to de-emphasize target discovery in favour of biology-driven lead generation - is in a way a "return to the fundamentals of drug discovery"10, the historically validated use of biology itself to discover bioactive compounds. Clearly there are disease processes that cell systems biology cannot at present model, but cell systems approaches should lend themselves readily to drug discovery in inflammation and immune biology, in cardiovascular, metabolic and bone diseases, and in the pathobiology of cell proliferation and cancer. The application of complex biology through human cell systems approaches could lead to a more efficient design for the future of drug discovery.

Eugene C. Butcher is in the Department of Pathology, Stanford University, Stanford, California 94305-5324, USA. e-mail: ebutcher@stanford.edu doi: $10.1038 / \mathrm{nrd} 1754$

Published online 24 May 2005

1. Horrobin, D. F. Modern biomedical research: an internally self-consistent universe with little contact with medical reality? Nature Rev. Drug Discov. 2, 151-154 (2003).

2. DiMasi, J. A., Hansen, R. W. \& Grabowski, H. G. The price of innovation: new estimates of drug development costs. J. Health Econ. 22, 151-185 (2003).

3. Drews, J. Strategic trends in the drug industry. Drug Discov. Today 8, 411-420 (2003).

4. Horrobin, D. F. Realism in drug discovery - could Cassandra be right? Nature Biotechnol. 19, 1099-1100 (2001).

5. Kubinyi, H. Drug research: myths, hype and reality. Nature Rev. Drug Discov. 2, 665-668 (2003).

6. Goodall, S., Ringel, M. \& Tollman, P. Rising to the Productivity Challenge: a Strategic Framework for Biopharma [online], < http://www.bcg.com/publications/ files/Rising_to_the_Productivity_Challenge.pdf > (Boston Consulting Group, 2004).
7. Zambrowicz, B. P. \& Sands, A. T. Knockouts model the 100 best-selling drugs - will they model the next 100 ? Nature Rev. Drug Discov. 2, 38-51 (2003).

8. Hopkins, A. L. \& Groom, C. R. The druggable genome. Nature Rev. Drug Discov. 1, 727-730 (2002).

9. Williams, M. Target validation. Curr. Opin. Pharmacol. 3 571-577 (2003).

10. Williams, M. A return to the fundamentals of drug discovery? Curr. Opin. Investig. Drugs 5, 29-33 (2004).

1. Mestas, J. \& Hughes, C. C. Of mice and not men: differences between mouse and human immunology. J. Immunol. 172, 2731-2738 (2004).

12. Ma, P. \& Zimmel, R. Value of novelty? Nature Rev. Drug Discov. 8, 571-572 (2002).

13. Bhadriraju, K. \& Chen, C. S. Engineering cellular microenvironments to improve cell-based drug testing. Drug Discov. Today 7, 612-620 (2002).

14. Kunkel, E. J. et al. An integrative biology approach for analysis of drug action in models of human vascular inflammation. FASEB J. 18, 1279-1281 (2004).

15. Butcher, E. C., Berg, E. L. \& Kunkel, E. J. Systems biology in drug discovery. Nature Biotechnol. 22, 1253-1259 (2004)

16. Liu, G. et al. Novel p-arylthio cinnamides as antagonists of leukocyte function-associated antigen-1/intracellular adhesion molecule-1 interaction. 2. Mechanism of inhibition and structure-based improvement of pharmaceutical properties. J. Med. Chem. 44 1202-1210 (2001).

17. Domino, E. F. History of modern psychopharmacology: a personal view with an emphasis on antidepressants. Psychosom. Med. 61, 591-598 (1999).

18. Kunkel, E. J. et al. Rapid structure-activity and selectivity analysis of kinase inhibitors by BioMAP analysis in complex human primary cell-based models. Assay Drug Dev. Technol. 2, 431-441 (2004).

19. Lipinski, C. A., Lombardo, F., Dominy, B. W. \& Feeney, P. J. Experimental and computational approaches to estimate solubility and permeability in drug discovery and development settings. Adv. Drug Deliv. Rev. 46, 3-26 (2001).
20. MacCoss, M. \& Baillie, T. A. Organic chemistry in drug discovery. Science 303, 1810-1813 (2004).

21. Giuliano, K. A., Haskins, J. R. \& Taylor, D. L. Advances in high content screening for drug discovery. Assay Drug Dev. Technol. 1, 565-577 (2003).

22. Gunther, E. C., Stone, D. J., Gerwien, R. W., Bento, P. \& Heyes, M. P. Prediction of clinical drug efficacy by classification of drug-induced genomic expression profiles in vitro. Proc. Natl Acad. Sci. USA 100, 9608-9613 (2003).

23. Bovet, D. The relationships between isoterism and competitive phenomena in the field of drug therapy of the autonomic nervous system and that of the neuromuscular transmission (Nobel lecture] [online] <http://nobelprize.org/medicine/laureates/1957/bovetlecture.pdf> (1957)

24. Godl, K. et al. An efficient proteomics method to identify the cellular targets of protein kinase inhibitors. Proc. Nat Acad. Sci. USA 100, 15434-15439 (2003).

25. US Department of Human and Health Services: US Food and Drug Administration. Innovation or Stagnation: Challenge and Opportunity on the Critical Path to New Medical Products [online],

<http://www.fda.gov/oc/initiatives/criticalpath/ whitepaper.html> (2004)

\section{Acknowledgments}

The author thanks M. Williams (Northwestern University, llinois) H. Kubinyi (Weisenheim am Sand, Germany), E. Berg and E. Kunkel (Bioseek, Inc.) for critical review of the manuscript.

Competing interests statement

The author declares competing financial interests: see Web version for details.

\section{FURTHER INFORMATION}

Butcher laboratory: http://butcherlab.stanford.edu/

Access to this interactive links box is free online.

\title{
A pathophysiological paradigm for the therapy of psychiatric disease
}

\author{
Michael Spedding*, Thérèse Jay*, Jorge Costa e Silva` and Laurent Perret ${ }^{\star}$
}

Abstract | Despite enormous progress in fundamental knowledge in neuroscience, no revolutionary therapies in psychiatry (and neurology) have emerged in the past ten years. Most drugs alleviate symptoms, rather than restoring the 'set point' of brain function from a pathological position to a more normal one. We propose a hypothesis-driven, systems-level approach to drug discovery and development that is based on pathophysiology and which uses new animal models.

Psychiatric diseases continue to create conspicuous social problems, not least among young people, a group in which there has been a steady increase in rates of depression, suicide and psychosocial disorders in the past half-century. But despite the phe- nomenal growth in the last decade in our understanding of brain mechanisms, there have been no major breakthroughs in the treatment of psychiatric and neurological diseases - drug discovery seems to be in a crisis, with no major new drugs registered. Why has the industry been so ineffective (BOX 1)?

Two reviews in this journal have addressed this drug-discovery crisis, arguing that genomic targets might help the discovery of new antidepressants ${ }^{1}$ and that 'selectively' non-selective drugs will form the basis of new antipsychotics ${ }^{2}$. Nevertheless, disorders such as depression have clear system dysfunctions, involving distinct brain areas ${ }^{3-7}$, which can be modelled in animals. Although these disorders have genetic, developmental and environ- 